\title{
Random registry shifts in quasi-one-dimensional adsorbate systems
}

\author{
J. Schäfer, ${ }^{1,2}$ S. C. Erwin, ${ }^{3}$ M. Hansmann, ${ }^{4}$ Z. Song, ${ }^{4}$ E. Rotenberg, ${ }^{2}$ S. D. Kevan, ${ }^{5}$ C. S. Hellberg, ${ }^{3}$ and K. Horn ${ }^{4}$ \\ ${ }^{1}$ Institut für Physik, Universität Augsburg, 86135 Augsburg, Germany \\ ${ }^{2}$ Advanced Light Source, Lawrence Berkeley National Laboratory, Berkeley, California 94720 \\ ${ }^{3}$ Center for Computational Materials Science, Naval Research Laboratory, Washington DC 20375 \\ ${ }^{4}$ Fritz-Haber-Institut der Max-Planck-Gesellschaft, Faradayweg 4-6, 14195 Berlin, Germany \\ ${ }^{5}$ Department of Physics, University of Oregon, Eugene, Oregon 97403
}

(Received 13 November 2002; published 18 February 2003)

\begin{abstract}
The apparent contradiction of one-dimensional adsorbate chains on $\mathrm{Si}(111)$ having a $3 \times 2$ unit cell and yet a $3 \times 1$ diffraction pattern is resolved for the example of $\mathrm{Ba} / \mathrm{Si}(111)-(3 \times 2)$. Random registry shifts between adsorbate chains are observed in tunneling microscopy, with very short interchain correlation lengths. Fourier analysis provides a natural explanation for a pseudo- $(3 \times 1)$ diffraction pattern. Within density-functional theory such registry shifts can occur with essentially negligible energy cost, leading to entropy-driven, virtually perfect disorder. Substrate states of high symmetry and one-dimensional character are inferred to promote this phenomenon.
\end{abstract}

DOI: 10.1103/PhysRevB.67.085411 PACS number(s): 73.20. $-\mathrm{r}, 68.65 .-\mathrm{k}, 68.35 .-\mathrm{p}$, 71.15. $-\mathrm{m}$

\section{INTRODUCTION}

Semiconductor surface reconstructions induced by the adsorption of metals provide unique opportunities for studying interactions in quasi-one-dimensional (1D) systems. A longstanding question for many such structures on $\mathrm{Si}(111)$ is the discrepancy between scanning-tunneling microscopy (STM) and low-energy electron-diffraction (LEED) images: STM shows " $\times 2$ " periodicity along the adsorbate chains, while LEED yields " $\times 1$ " periodicity, in some cases with faint half-order streaks. Examples include three classes of systems on $\mathrm{Si}(111)$ : (i) the $(3 \times 2)$ reconstructions induced by the alkaline-earth metals $\mathrm{Mg}, \mathrm{Ca}$, and $\mathrm{Ba}^{1-4}$ (ii) the In-induced $(8 \times 2)$ reconstruction; ${ }^{5}$ and (iii) the Au-induced $(5 \times 2)$ reconstruction. ${ }^{6,7}$ All of these systems feature linear arrangements of adsorbates with local positional degeneracy. As a result, the phase correlation between adsorbate chains directly reflects the competition between the energy cost from interchain interactions and the energy gain from entropy. The outcome of this competition can greatly affect the effective periodicities and zone boundaries in real and reciprocal space, respectively. A particularly striking example of this is the Ba-induced $\mathrm{Si}(111)$ reconstruction, which appears (3 $\times 2)$ in STM despite missing or very faint half-order LEED streaks. $^{8-10}$

In this paper we use $\mathrm{Ba} / \mathrm{Si}(111)-(3 \times 2)$ as a model system for the investigation of phase correlations between $\mathrm{Ba}$ chains to explain the apparent discrepancy between LEED and STM periodicities. First, we present an analysis of the local chain structure based on STM data, from which we identify a high density of phase slips. Using a scattering simulation, we demonstrate that random registry shifts between well-ordered chains are the principal mechanism responsible for the pseudo- $(3 \times 1)$ LEED pattern. Second, we explore the energetics of such phenomena by estimating the energy scale for registry shifts within density-functional theory (DFT), assuming a recently proposed structural model for $\mathrm{Ba}(3 \times 2) .{ }^{9}$ Our theoretical results confirm a nearly negligible cost for registry shifts of individual chains. Finally, we show that a comparison of our calculated electronic band structure to angle-resolved photoemission spectroscopy (ARPES) data is consistent with twofold adsorbate positional degeneracy together with 1D electronic character. We argue that these are general criteria for phase slips, and are likely to be applicable to other " $\times 2$ " adsorbate systems on $\operatorname{Si}(111)$.

\section{EXPERIMENT AND STRUCTURAL OVERVIEW}

Experimentally, single-domain samples were prepared in ultrahigh vacuum on vicinal $\operatorname{Si}(111)$ wafers (miscut $2^{\circ}$ towards $[11 \overline{2}]$ ). Ba metal was deposited from a getter source onto the substrate held at room temperature. Subsequent annealing at $\sim 1200 \mathrm{~K}$ for several seconds yielded a very sharp, single-domain $(3 \times 1)$ LEED image as shown in Fig. 1(a).

The STM data in Fig. 1(b) show empty-state topographic data of a representative part of the surface, recorded at room temperature. The presence of $3 \times 2$ unit cells is readily visible, in apparent contradiction to the LEED image of Fig. 1(a). The existence of a $3 \times 2$ structure has been noted earlier. ${ }^{9}$ Closer analysis, however, reveals that the surface mesh is not consistent with a simple extended $3 \times 2$ array of unit cells. Instead, we observe changes in interchain alignment [from "up" to "down" in Fig. 1(b)], corresponding to a phase shift of half a unit cell, i.e., by " $\times 1$." These phase shifts are very frequent and occur essentially at random. The image also shows a defect where a phase shift occurs within the same chain. The intensity maxima in the STM images are tentatively assigned to $\mathrm{Ba}$ atoms, although the technique provides no straightforward identification. ${ }^{6}$ Further below from the structural model it emerges that the assignment is reasonably justified. A defect in the chain sequence in this interpretation may thus be called a Ba vacancy. From counting cells in the images, or equivalently from autocorrelation plots we obtain information on the correlation lengths. Along the chains, it is rather large (typically $>20 \mathrm{Ba}$ atoms), while across the chains shifts occur typically after just two to three chains. 
a)

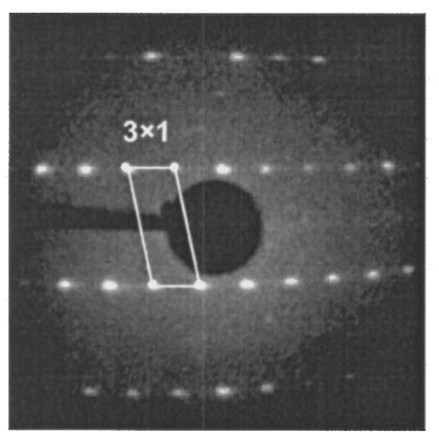

b)

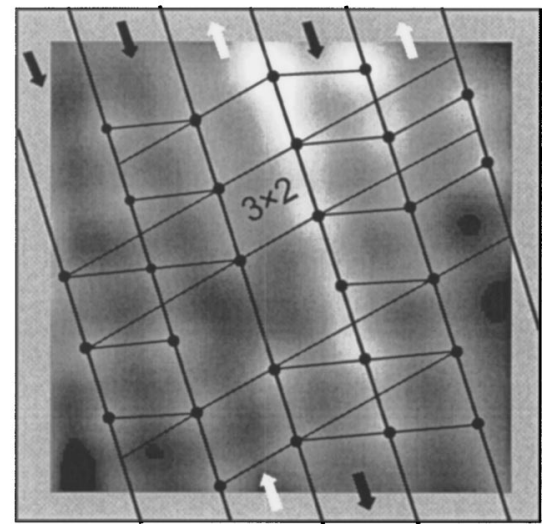

FIG. 1. (a) Ba-Si(111) LEED image taken with 84-eV electrons. The periodicity corresponds to $3 \times 1$, and within detection limit no half order is observed. (b) Empty-state STM image of same reconstruction $\left(48 \times 48 \AA, U_{s}=2.0 \mathrm{~V}\right)$. Local geometry is $3 \times 2$, contrary to LEED. Registry shifts between chains in discrete half-cell steps are very common. They may originate from defects (e.g., of a Ba atom) on the chain.

\section{SCATTERING SIMULATION}

The implication of such phase shifts (as opposed to simple defects) for LEED measurements is easily demonstrated in a scattering simulation. We have used a square area of $5 \times 10^{3}$ unit cells with $(3 \times 2)$ dimension, each containing a delta-function scattering potential to represent a Ba atom. The phase shifts observed in STM are modeled as random registry shifts (RRS). This model assumes (i) an infinite correlation length along the chains (only limited by the cluster size), and (ii) a random-phase correlation between the chains. The model can only give qualitative information regarding LEED intensities, yet will correctly reflect the symmetries originating from long-range order of the unit cells-or the absence thereof.

The Fourier transform of the simulated surface, along with that of the STM data, are plotted as a power spectrum (the square of the absolute intensities, as in a diffraction experiment) in Fig. 2. Both simulation and STM data have a missing $3 \times 2$ reflection. The intensity is smeared out between these locations. Any STM Fourier transform suffers from a decaying frequency envelope originating from the limited area transformed, so higher-order reflections are not visible. The large-area simulation shows very clearly the second order of reflections. The streaking can be understood as

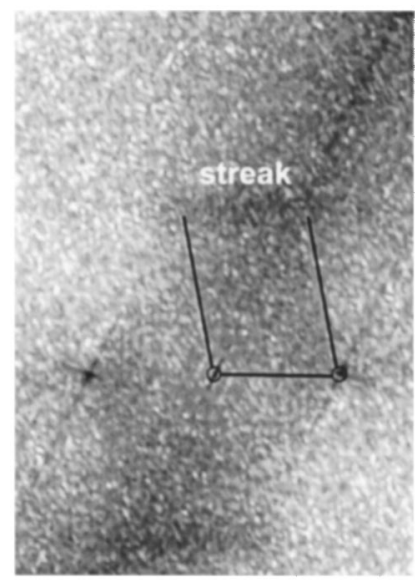

a) FT-STM

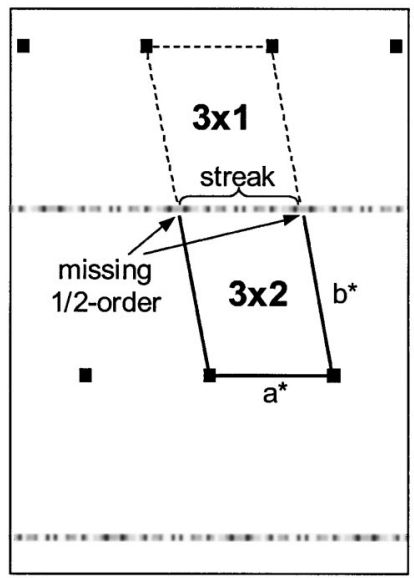

b) Simulation
FIG. 2. (a) Power spectrum obtained from a large-scale STM image $(340 \times 280 \AA)$. A half-order reflex, typical for $3 \times 2$, is missing. Horizontal streaks are observed instead (diagonal lines are a finite-size fast Fourier-transform artifact). (b) Power spectrum of simulated $(3 \times 2)$ structure with RRS. Absence of a half order is confirmed, and a pseudo- $(3 \times 1)$ remains. Peak intensities in a streak are typically $1 \%-5 \%$ of the $(3 \times 1)$ peaks, and are scaled to full gray scale for display purposes.

resulting from $3 \times 1$ units in the presence of $N \times 2$ superstructures of varying width $N$ across the chains (see Fig. 3 below). Every other line of reflections is thus smeared out sideways, because $1 / N$ varies randomly.

The simulated streak intensity is about two orders-ofmagnitude smaller than the main spots. Our own work does not resolve a streak in between $(3 \times 1)$ spots. Okuda et al. ${ }^{10}$ do obtain a very faint streak. For Ca- $(3 \times 2)$ LEED data ${ }^{11}$ weak half-order streaks are reported also. This confirms the low intensity of this feature at detection threshold. Surface imperfections will contribute additional background. Also, the degree of randomness of the chain correlation may depend on particular preparation details. The overall result is a $3 \times 1$ LEED pattern, originating from random registry shifts of the $\mathrm{Ba}$ chains. This finding reconciles the microscopic unit cell with the macroscopic diffraction pattern.

\section{STRUCTURE MODEL AND ENERGETICS}

\section{A. Density-functional theory}

In order to estimate the energy scale for such registry shifts, we refer to the structural model for $\mathrm{Ba} / \mathrm{Si}(111)$ $(3 \times 2)$ recently proposed by Lee et al. ${ }^{9}$ This model is closely related to the simpler $(3 \times 1)$ reconstruction of $\mathrm{Si}$ (111) induced by alkali metals, in which linear honeycomblike arrangements of top-layer Si atoms form channels where chains of alkali adsorbates reside. This "honeycombchain channel" (HCC) reconstruction is stabilized by electron donation from the alkalis into bonding combinations of Si surface orbitals. ${ }^{12,13}$ A period-doubled variant of this HCC reconstruction can be induced by divalent adsorbates such as $\mathrm{Ba}$ and other alkaline-earth metals. Electron counting then requires half the adsorbate coverage, with a $\mathrm{Ba}$ atom in every 


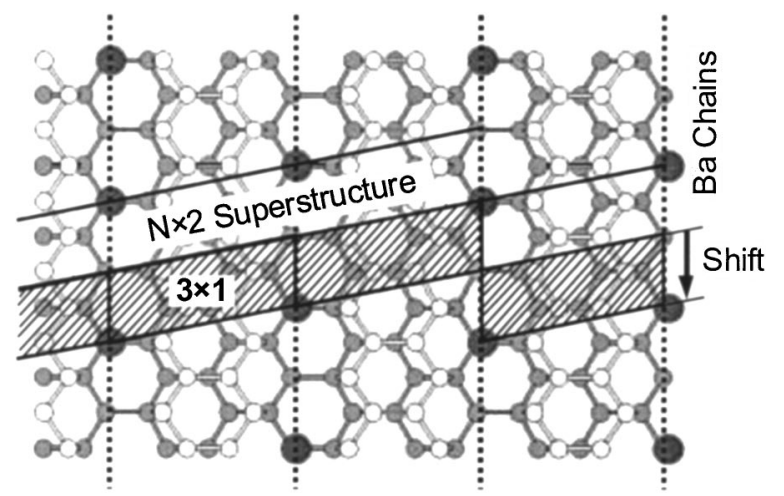

FIG. 3. LDA structural model for modified $3 \times 2$ reconstruction. Between neighboring chains, a registry shift by half a unit cell is energetically possible, without change in the individual chain structure. This leads to $N \times 2$ superstructures of varying width $N$. This mechanism exploits a substrate with doubled periodicity.

second $(3 \times 1)$ substrate cell. ${ }^{9}$ This leads to a doubling of the adsorbate spacing within each chain, with only minor perturbation of the underlying $(3 \times 1)$ substrate. Thus, in the absence of interchain interactions, each chain would exhibit a twofold local positional degeneracy.

We use DFT in the local-density approximation (LDA) to study the energetics of registry shifts of the Ba chains. We model the surface using an $H$-terminated slab with eight layers of Si plus the reconstructed Si and adsorbed Ba layers. Total energies and forces were calculated using ultrasoft pseudopotentials within VASP; ${ }^{14}$ the kinetic-energy cutoff was $150 \mathrm{eV}$ and four irreducible $k$ points were used to sample the surface Brillouin zone. We start from the basic structural model suggested by Lee et al. ${ }^{9}$ By relaxing this model under the assumption of $3 \times 2$ periodicity, we find that $\mathrm{Ba}$ adsorbates are stable at both the $\mathrm{H}_{3}$ and $T_{4}$ surface sites, with the $T_{4}$ site preferred by $35 \mathrm{meV}$ per Ba. This is much larger than the energy scale for registry shifts, so we assume that all such shifts leave the Ba adsorbates in $T_{4}$ sites. Furthermore, we simulate the shifts as rigid translations of the Ba chains on a rigid $\mathrm{Si}(3 \times 1)$ substrate, which we take to be the appropriate average of the relaxed $(3 \times 2)$ substrate coordinates. For the configuration of registry shifts illustrated in Fig. 3, we find that the DFT total energy is changed by less than $0.1 \mathrm{meV}$ per $\mathrm{Ba}$, relative to the unshifted $(3 \times 2)$ configuration. This result suggests that the energy scale for registry shifts is far below room temperature.

A simple model explains this very small energy scale. The $\mathrm{Ba}$ adsorbates are +2 ions, and the large distance between them justifies representing them as point charges. In the absence of substrate-mediated interactions, the only $\mathrm{Ba}-\mathrm{Ba}$ interactions are electrostatic. Within this approximation, the total energy of any configuration of chains is given simply by its Madelung energy. The underlying $3 \times 1$ symmetry implies that the nearest-neighbor contribution to the Madelung sum is the same for all configurations. This degeneracy is broken only at the next-nearest neighbor, which partially explains the small energy scale. Indeed, for the configuration of Fig. 3 , the electrostatic energy cost relative to the unshifted configuration is only $0.003 \mathrm{meV}$ per $\mathrm{Ba}$. Other processes that might also destroy the long-range $3 \times 1$ order are $\mathrm{Ba}-\mathrm{Si}$ exchanges. There are various configurations possible, which will lead to a local defect. Such defects are relatively abundant, as seen, e.g., in the STM image Fig. 1(b). However, such additional perturbations do not affect the arguments given for the exceptionally low-energy cost of rigid chain shifts.

An important question is whether simple surface diffusion can lead to the observed registry shifts. On a surface without defects, no shifts are possible because the energy barrier is infinite. In the presence of defects, finite chains will experience a barrier of approximately $M \cdot \Delta E$, where $M$ is the number of atoms in the chain; within the LDA we find $\Delta E$ $=140 \mathrm{meV}$. Thus for typical attempt frequencies of $10^{13} \mathrm{~s}^{-1}$, we predict that chains of length $M=22$ will shift on average once per second at the annealing temperature of $1200 \mathrm{~K}$. Chains of this length are consistent with the correlation lengths we obtained in Sec. II.

\section{B. Electronic structure}

Random registry shifts have profound consequences for the electronic properties of the surface. Here we demonstrate this by comparing our theoretical band structure with our photoemission data. Previously published photoemission data ${ }^{10}$ were taken before a structural model was clearly established. The data from that study indicate $(3 \times 1)$ symmetry, but do not test for a repetition of that periodicity in higher surface Brillouin zones. We have collected ARPES data at beamline 7.0.1 of the Advanced Light Source, Berkeley (energy resolution $\sim 70 \mathrm{meV}$, angular resolution $\pm 0.4^{\circ}$ ). Figure $4(\mathrm{a})$ shows the valence bands in the second $(3 \times 1)$ surface Brillouin zone, which establishes the repetition of the surface band set. Extension of these surface bands clearly beyond the $(3 \times 2)$ zone boundary indicates that this zone boundary is essentially lifted.

The weakness of the $(3 \times 2)$ zone boundary could, in principle, be confirmed theoretically by unfolding the calculated $(3 \times 2)$ band structure into a $(3 \times 1)$ zone. The $(3$ $\times 2$ ) surface bands of interest mostly fall within the projected bulk bands, making an unambiguous unfolding impossible. Instead we approximate the unfolding by calculating bands for an isoelectronic system $\mathrm{Li} / \mathrm{Si}(111)-(3 \times 1)$, using the atomic coordinates obtained from $\mathrm{Ba} / \mathrm{Si}(111)-(3 \times 2)$ (suitably averaged along the chain direction). The resulting bands in Fig. 4(b) do not differ significantly from the alkali case, and are in excellent correspondence with the ARPES data. The calculated dispersion perpendicular to the chain direction (not shown) is very weak, in good agreement with the 1D character of the data shown in Fig. 4(c).

From this comparison we confirm two key aspects of our description: (i) the $(3 \times 1)$ physical and electronic natures of the substrate, both of which are largely unaffected by the $(3 \times 2)$ adsorbate periodicity; and (ii) the highly 1D character of the occupied states. The clear implications are that the $(3 \times 1)$ HCC cells are invariant against shifts of the adsorbate chain, and that the chains are isolated sufficiently 

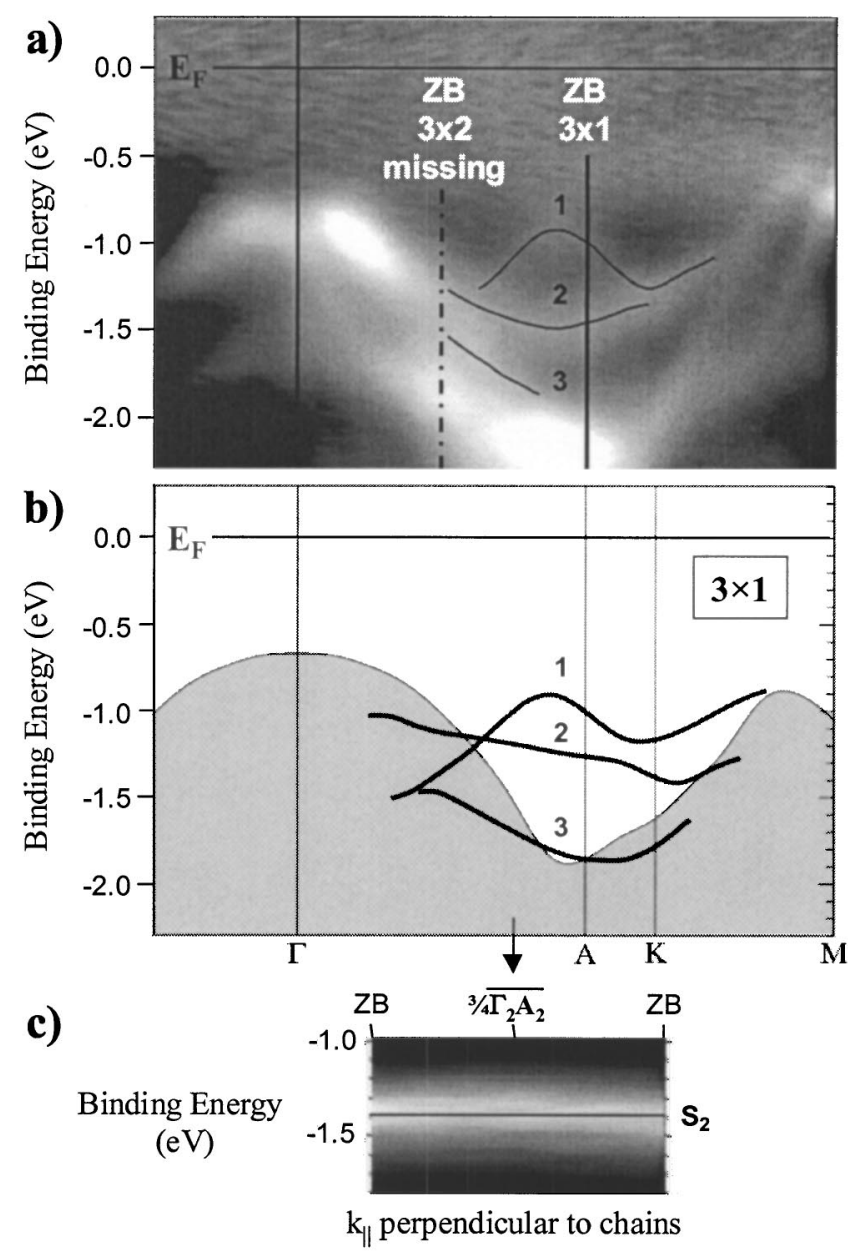

FIG. 4. (a) Band structure in second surface Brillouin zone ( $T$ $=150 \mathrm{~K}, h \nu=90 \mathrm{eV}$, spectra normalized to their average). No 3 $\times 2$ zone boundary (ZB) is observed, instead symmetry is $3 \times 1$. (b) LDA band structure in extended zone scheme, using a $(3 \times 1)$ unit cell with atomic positions derived from $\mathrm{Ba}(3 \times 2)$. Surface bands $1-3$ are reidentified in ARPES. (c) Lack of dispersion in ARPES perpendicular to chains, taken at $0.75 \bar{\Gamma}-\bar{A}$.

well that they do not experience any lateral interaction. These two criteria underlie the negligible energy cost of registry shifts.

\section{DISCUSSION AND SUMMARY}

Loss of phase coherence in quasi-1D adsorbate systems should be expected in a variety of analogous systems includ- ing, of course, $\mathrm{Ca}$ - and $\mathrm{Mg}$-induced $\mathrm{Si}(111)-(3 \times 2)$ with the same HCC substrate. ${ }^{15,16}$ For the quasi-1D system In/Si(111)- $(8 \times 2)$, previously explored by us and others, ${ }^{17}$ similar arguments will hold. Kumpf et al. ${ }^{5}$ have proposed that the observed low-temperature In- $(8 \times 2)$ structure does not originate from a charge-density wave, but rather from a modification of the reconstruction. Their x-ray-diffraction data contain streaks similar to our simulated random registry shifts. Regardless of the detailed mechanism leading to $\times 2$ periodicity, the twofold positional degeneracy with respect to the substrate unit cell is an important common property. In general, random registry shifts will occur more easily if the electronic character is close to $1 \mathrm{D}$, so that negligible energy has to be expended for a lattice distortion. This criterion appears to be fulfilled in these systems. For example, Yeom et $a l .{ }^{17}$ have demonstrated the 1D character of In on $\mathrm{Si}$, and a $\times 1$ substrate with a modified HCC cell has been recently discussed to explain it. ${ }^{18}$ The random-registry-shift mechanism appears to provide a natural explanation for the pseudo $\times 1$ LEED pattern of these classes of reconstruction as well. For rare-earth metals on $\mathrm{Si}(111)$, such as $\mathrm{Sm}$ and $\mathrm{Yb}$, similarly a local $3 \times 2$ reconstruction had been found, while LEED yields a $3 \times 1$ pattern with half-order streaks. ${ }^{19}$ The extent to which the system is one dimensional electronically remains to be investigated. For all these adsorbate reconstructions, the criterion of rigidity of the substrate against translations of the adsorbates further lowers the threshold for registry shifts. This aspect-demonstrated in our DFT calculation-contributes to the virtually perfect randomness in the $\mathrm{Ba} / \mathrm{Si}(111)-(3 \times 2)$ system.

In summary, we have shown that the Ba-induced pseudo$(3 \times 1)$ reconstruction of $\mathrm{Si}(111)$ is highly susceptible to registry shifts. Those phase fluctuations occur because the silicon substrate reconstruction is of higher symmetry than the adsorbate unit cell, so that the system can lower its total energy through entropy. Random registry shifts then provide an explanation as to why the local $3 \times 2$ structure leads to a global $3 \times 1$ diffraction pattern. The study exemplifies frozen-in structural fluctuations in highly 1D confined systems.

\section{ACKNOWLEDGMENTS}

This work was supported by the DOE under Grant No. DE-FG06-86ER45275. S.C.E. and C.S.H. acknowledge support from the ONR and a grant of HPC time from the DOD MSRC ASCWP.
${ }^{1}$ M. A. Olmstead, R. I. G. Uhrberg, R. D. Bringans, and R. Z. Bachrach, J. Vac. Sci. Technol. B 4, 1123 (1986).

${ }^{2}$ J. Quinn and F. Jona, Surf. Sci. 249, L307 (1991).

${ }^{3}$ H. H. Weitering, Surf. Sci. 355, L271 (1996).

${ }^{4}$ O. Kubo, A. A. Saranin, A. V. Zotov, J.-T. Ryu, H. Tani, T. Harada, M. Katayama, V. G. Lifshits, and K. Oura, Surf. Sci. 415, L971 (1998).
${ }^{5}$ C. Kumpf, O. Bunk, J. H. Zeysing, Y. Su, M. Nielsen, R. L. Johnson, R. Feidenhansl, and K. Beechgard, Phys. Rev. Lett. 85, 4916 (2000).

${ }^{6}$ R. Losio, K. N. Altmann, and F. J. Himpsel, Phys. Rev. Lett. 85, 808 (2000).

${ }^{7}$ I. G. Hill and A. B. McLean, Phys. Rev. B 55, 15664 (1997).

${ }^{8}$ H. H. Weitering, Surf. Sci. Lett. 355, L271 (1996). 
${ }^{9}$ G. Lee, S. Hong, H. Kim, D. Shin, J.-Y. Koo, H.-I. Lee, and D. W. Moon, Phys. Rev. Lett. 87, 056104 (2001).

${ }^{10}$ T. Okuda, H. Ashima, H. Takeda, K.-S. An, A. Harasawa, and T. Kinoshita, Phys. Rev. B 64, 165312 (2001).

${ }^{11}$ A. A. Saranin, V. G. Lifshits, K. V. Ignatovich, H. Bethge, R. Kayser, H. Goldbach, A. Klust, J. Wollschläger, and M. Henzler, Surf. Sci. 448, 87 (2000).

${ }^{12}$ S. C. Erwin and H. H. Weitering, Phys. Rev. Lett. 81, 2296 (1998)

${ }^{13}$ M.-H. Kang, J.-H. Kang, and S. Jeong, Phys. Rev. B 58, 13359 (1998).

${ }^{14}$ G. Kresse and J. Hafner, Phys. Rev. B 47, R558 (1993); G. Kresse and J. Furthmüller, ibid. 54, 11169 (1996).
${ }^{15}$ A. A. Baski, S. C. Erwin, M. S. Turner, K. M. Jones, J. W. Dickinson, and J. A. Carlisle, Surf. Sci. 476, 22 (2001).

${ }^{16}$ K. S. An, R. J. Park, J. S. Kim, C. Y. Park, C. Y. Kim, J. W. Chung, T. Abukawa, S. Kono, T. Kinoshita, A. Kakizaki, and T. Ishii, Surf. Sci. 337, L789 (1995).

${ }^{17}$ H. W. Yeom, S. Takeda, E. Rotenberg, I. Matsuda, K. Horikoshi, J. Schäfer, C. M. Lee, S. D. Kevan, T. Ohta, T. Nagao, and S. Hasegawa, Phys. Rev. Lett. 82, 4898 (1999).

${ }^{18}$ J.-H. Cho, D.-H. Oh, K. S. Kim, and L. Kleinman, Phys. Rev. B 64, 235302 (2001).

${ }^{19}$ C. Wigren, J. N. Andersen, R. Nyholm, M. Göthelid, M. Hammar, C. Törnevik, and U. O. Karlsson, Phys. Rev. B 48, 11014 (1993). 